W. M. ZAJĄCZKOWSKI (Warszawa)

\title{
SOME STABILITY PROBLEM FOR THE NAVIER-STOKES EQUATIONS IN THE PERIODIC CASE
}

Abstract. The Navier-Stokes motions in a box with periodic boundary conditions are considered. First the existence of global regular twodimensional solutions is proved. Since the external force does not decay in time, the solution has the same property. The necessary estimates and existence are proved step by step in time. Dissipation in the Navier-Stokes equations makes this approach possible. Assuming that the initial velocity and the external force are sufficiently close to the initial velocity and the external force of the two-dimensional problems we prove existence of global three-dimensional regular solutions which remain close to the twodimensional solutions for all time.

1. Introduction. The aim of this paper is to prove stability of twodimensional periodic solutions in the set of three-dimensional periodic solutions to the Navier-Stokes equation. We consider the three-dimensional fluid motions in the box $\Omega=[0, L]^{3}, L>0$, described by

$$
\begin{array}{ll}
v_{t}+v \cdot \nabla v-\nu \Delta v+\nabla p=f & \text { in } \Omega \times \mathbb{R}_{+}, \\
\operatorname{div} v=0 & \text { in } \Omega \times \mathbb{R}_{+}, \\
\left.v\right|_{t=0}=v(0) & \text { in } \Omega,
\end{array}
$$

where $v=\left(v_{1}(x, t), v_{2}(x, t), v_{3}(x, t)\right) \in \mathbb{R}^{3}$ is the velocity of the fluid, $x=$ $\left(x_{1}, x_{2}, x_{3}\right)$ with $x_{i} \in(0, L), i=1,2,3$, is a given Cartesian system of coordinates, $p=p(x, t) \in \mathbb{R}$ is the pressure and $f=\left(f_{1}(x, t), f_{2}(x, t), f_{3}(x, t)\right) \in \mathbb{R}^{3}$ is the external force field. Finally, $\nu>0$ is the constant viscosity coefficient and the dot denotes the scalar product in $\mathbb{R}^{3}$.

2010 Mathematics Subject Classification: 35Q30, 76D05, 76N10, 35B35, 76D03.

Key words and phrases: incompressible Navier-Stokes equations, stability of twodimensional solutions, global regular solutions, periodic boundary conditions.

Received 15 June 2016; revised 30 August 2018.

Published online 23 April 2019. 
Two-dimensional solutions to (1.1) are such that $v=v_{s}=\left(v_{s 1}\left(x_{1}, x_{2}, t\right)\right.$, $\left.v_{s 2}\left(x_{1}, x_{2}, t\right), 0\right) \in \mathbb{R}^{2}, \quad p=p_{s}\left(x_{1}, x_{2}, t\right) \in \mathbb{R}, f=f_{s}=\left(f_{s 1}\left(x_{1}, x_{2}, t\right)\right.$, $\left.f_{s 2}\left(x_{1}, x_{2}, t\right), 0\right) \in \mathbb{R}^{2}$; they satisfy the problem

$$
\begin{array}{ll}
v_{s t}+v_{s} \cdot \nabla v_{s}-\nu \Delta v_{s}+\nabla p_{s}=f_{s} & \text { in } \Omega \times \mathbb{R}_{+}, \\
\operatorname{div} v_{s}=0 & \text { in } \Omega \times \mathbb{R}_{+}, \\
\left.v_{s}\right|_{t=0}=v_{s}(0) & \text { in } \Omega .
\end{array}
$$

We introduce the quantities

$$
u=v-v_{s}, \quad q=p-p_{s},
$$

which are solutions to the problem

$$
\begin{array}{ll}
u_{t}+u \cdot \nabla u-\nu \Delta u+\nabla q=-v_{s} \cdot \nabla u-u \cdot \nabla v_{s}+g & \text { in } \Omega \times \mathbb{R}_{+}, \\
\operatorname{div} u=0 & \text { in } \Omega \times \mathbb{R}_{+}, \\
\left.u\right|_{t=0}=u(0) & \text { in } \Omega,
\end{array}
$$

with $g=f-f_{s}$. To show stability of solutions to (1.2) we need to prove smallness of the quantities (1.3) in some norms for all $t \in \mathbb{R}_{+}$. For this purpose we apply the energy method. For this we need the Poincaré inequality. Since it does not hold for solutions to problems 1.2 or $(1.4)$, we introduce the quantities

$$
\begin{array}{ccc}
\bar{v}_{s}=v_{s}-f_{\Omega} v_{s} d x, & \bar{p}_{s}=p_{s}-\int_{\Omega} p_{s} d x, & \bar{f}_{s}=f_{s}-f_{s} d x, \\
\bar{u}=u-\int_{\Omega} u d x, & \bar{q}=q-\int_{\Omega} q d x, & \bar{g}=g-\int_{\Omega} g d x,
\end{array}
$$

where

$$
f_{\Omega} \omega d x=\frac{1}{|\Omega|} \int_{\Omega} \omega d x \quad \text { and } \quad|\Omega|=L^{3} .
$$

Applying the mean operator to $(1.2)_{1}$ and $(1.4)_{1}$ and using the periodic boundary conditions we obtain

$$
\begin{aligned}
\frac{d}{d t} \int_{\Omega} v_{s} d x & =\int_{\Omega} f_{s} d x, \\
\frac{d}{d t} f_{\Omega} u d x & =\int_{\Omega} g d x .
\end{aligned}
$$

In view of (1.6), (1.7), and since any space derivative of the mean vanishes, we see that for the quantities (1.5) problems (1.2) and (1.4) take the forms

$$
\begin{array}{ll}
\bar{v}_{s t}+v_{s} \cdot \nabla \bar{v}_{s}-\nu \Delta \bar{v}_{s}+\nabla \bar{p}_{s}=\bar{f}_{s} & \text { in } \Omega \times \mathbb{R}_{+}, \\
\operatorname{div} \bar{v}_{s}=0 & \text { in } \Omega \times \mathbb{R}_{+}, \\
\left.\bar{v}_{s}\right|_{t=0}=\bar{v}_{s}(0) & \text { in } \Omega,
\end{array}
$$


and

$$
\begin{array}{ll}
\bar{u}_{t}+u \cdot \nabla \bar{u}-\nu \Delta \bar{u}+\nabla \bar{q}=-v_{s} \cdot \nabla \bar{u}-u \cdot \nabla \bar{v}_{s}+\bar{g} & \text { in } \Omega \times \mathbb{R}_{+}, \\
\operatorname{div} \bar{u}=0 & \text { in } \Omega \times \mathbb{R}_{+}, \\
\left.\bar{u}\right|_{t=0}=\bar{u}(0) & \text { in } \Omega .
\end{array}
$$

Now, we formulate the main results of this paper (for notation see Section 2). From Lemmas 3.1-3.3 we have

THEOREM 1 (two-dimensional solutions). Assume that for all $k \in \mathbb{N}_{0}$ and all $\sigma>3, \bar{f}_{s} \in L_{2}\left(k T,(k+1) T ; L_{\sigma}(\Omega)\right)$ and $\bar{v}_{s}(0) \in B_{\sigma, 2}^{1}(\Omega)$. Then there exists a solution $\left(\bar{v}_{s}, \bar{p}_{s}\right)$ to problem $(1.8)$ such that $\bar{v}_{s} \in W_{\sigma, 2}^{2,1}(\Omega \times(k T$, $(k+1) T))$ and $\nabla \bar{p}_{s} \in L_{2}\left(k T,(k+1) T ; L_{\sigma}(\Omega)\right)$ for all $k \in \mathbb{N}_{0}$, and we have the estimates

$$
\left\|\bar{v}_{s}\right\|_{W_{\sigma, 2}^{2,1}(\Omega \times(0, T))} \leq c\left(A^{2}+\left\|\bar{f}_{s}\right\|_{L_{2}\left(0, T ; L_{\sigma}(\Omega)\right)}+\left\|v_{s}(0)\right\|_{B_{\sigma, 2}^{1}(\Omega)}\right)
$$

and

$$
\left\|\bar{v}_{s}\right\|_{W_{\sigma, 2}^{2,1}(\Omega \times(k T,(k+1) T))} \leq c\left(A+A^{2}+\left\|\bar{f}_{s}\right\|_{L_{2}\left((k-1) T,(k+1) T ; L_{\sigma}(\Omega)\right)}\right)
$$

for all $k \in \mathbb{N}_{0}$, where

$$
\begin{aligned}
A=A_{5} & :=\left(1+\frac{c_{s 1}}{1-\exp \left(-\nu c_{s 1} T\right)}\right) A_{1}^{2}+\left\|v_{s x}(0)\right\|_{L_{2}}^{2}, \\
A_{1}^{2} & :=\frac{1}{\nu c_{s 1}} \sum_{k \in \mathbb{N}_{0}} \int_{k T}^{(k+1) T}\left\|\bar{f}_{s}(t)\right\|_{L_{2}}^{2} d t,
\end{aligned}
$$

and $c_{s 1}$ is the constant from the Poincaré inequality (2.3).

Lemma 4.2 implies

THEOREM 2 (stability). Let $c_{1}$ be the constant from the Poincaré inequality $(2.4), c_{4}=c_{4}\left(c_{1}\right)$ the constant from the embedding $c_{4}\|\bar{u}\|_{H 2} \leq\left\|u_{x x}\right\|_{L_{2}}$ (see (4.15) and (4.16)), and $c_{5}$ the constant from the interpolation $\left\|\bar{u}_{x}\right\|_{L_{3}} \leq$ $c_{5}\left\|\bar{u}_{x x}\right\|_{L_{2}}^{1 / 2}\left\|\bar{u}_{x}\right\|_{L_{2}}^{1 / 2}$ (after (4.15)). Let the assumptions of Theorem 1 hold. Suppose $\gamma \in\left(0, \gamma_{*}\right], \nu c_{4}-\left(c_{5} / \nu^{3}\right) \gamma_{*}^{2} \geq c_{*} / 2$ and $c_{*}<\nu c_{4}$. Assume that $\bar{g} \in C\left(\mathbb{R}_{+} ; L_{2}\right)$ and $\bar{u}(0) \in H^{1}$. Assume that

$$
\begin{aligned}
& \|\bar{u}(0)\|_{H^{1}}^{2} \leq \gamma \\
& G^{2}(t):=\frac{c_{5}}{\nu}\left[\left\|\bar{v}_{s x}\right\|_{L_{3}}^{2}\left|\int_{0}^{t} \int_{\Omega} g\left(x, t^{\prime}\right) d x d t^{\prime}+\int_{\Omega} u(0) d x\right|^{2}+\|\bar{g}\|_{L_{2}}^{2}\right] \leq c_{*} \frac{\gamma}{4},
\end{aligned}
$$


Let $T>0$ be given and $k \in \mathbb{N}_{0}$. Assume that

$$
\begin{aligned}
& \frac{c_{5}}{\nu} \int_{k T}^{(k+1) T}\left\|\bar{v}_{s x}\right\|_{L_{3}}^{2} d t \leq \frac{c_{*}}{4} T, \quad \int_{k T}^{(k+1) T} G^{2}(t) d t \leq \alpha \gamma, \\
& \alpha \exp \left(\frac{c_{*}}{4} T\right) \exp \left(-\frac{c_{*}}{4} T\right) \leq 1 .
\end{aligned}
$$

Then

$$
\|\bar{u}(t)\|_{H^{1}}^{2} \leq \gamma \quad \text { for } t \in \mathbb{R}_{+} .
$$

Finally, by the regularity theory for the Navier-Stokes equations we have

TheOREM 3. Let the assumptions of Theorems 1 and 2 hold. Then there exists a solution $(v, p)$ to problem (1.1) such that $v=v_{s}+u \in W_{2}^{2,1}(\Omega \times$ $(k T,(k+1) T))$ and $\nabla p=\nabla\left(p_{s}+q\right) \in L_{2}\left(k T,(k+1) T ; L_{2}(\Omega)\right)$ for all $k \in \mathbb{N}_{0}$, where $v_{s}, p_{s}, u$ are determined by Theorems 1 and 2 , respectively.

The first results connected with the stability of global regular solutions to the nonstationary Navier-Stokes equations were proved by Beirão da Veiga and Secchi [4], followed by Ponce, Racke, Sideris and Titi [17]. Paper [4] is concerned with the stability in $L_{p}$ norm of a strong three-dimensional solution of the Navier-Stokes system with zero external force in the whole space. In [17], assuming that the external force is zero and a three-dimensional initial function is close to a two-dimensional one in $H^{1}\left(\mathbb{R}^{3}\right)$, the authors showed the existence of a global strong solution in $\mathbb{R}^{3}$ which remains close to a twodimensional strong solution for all time. In [16] Mucha obtained a similar result under weaker assumptions about the smallness of the initial velocity perturbation.

In the class of weak Leray-Hopf solutions the first stability result was obtained by Gallagher [8]. She proved the stability of two-dimensional solutions of the Navier-Stokes equations with periodic boundary conditions under three-dimensional perturbations both in $L_{2}$ and $H^{1 / 2}$ norms.

The stability of nontrivial periodic regular solutions to the Navier-Stokes equations was studied by Iftimie [10] and by Mucha [14. The paper [14] is devoted to the case when the external force is a potential in $L_{r, \text { loc }}\left(\mathbb{T}^{3} \times[0, \infty)\right)$ and the initial data belongs to $W_{r}^{2-2 / r}\left(\mathbb{T}^{3}\right) \cap L_{2}\left(\mathbb{T}^{3}\right)$, where $r \geq 2$ and $\mathbb{T}$ is a torus. Under the assumption that there exists a global solution with data of regularity mentioned above and that small perturbations of data have the same regularity as above, the author proves that perturbations of the velocity and the gradient of the pressure remain small in $W_{r}^{2,1}\left(\mathbb{T}^{3} \times(k, k+1)\right)$ and $L_{r}\left(\mathbb{T}^{3} \times(k, k+1)\right), k \in \mathbb{N}_{0}$, respectively. Paper [10] contains results concerning stability of two-dimensional regular solutions to the Navier-Stokes system in a three-dimensional torus but here the initial data in the three-dimensional 
problem belongs to an anisotropic space of functions having different regularity in the first two directions than in the third direction, and the external force vanishes. Moreover, Mucha [15] studies the stability of regular solutions to the nonstationary Navier-Stokes system in $\mathbb{R}^{3}$ assuming that they tend in $W_{r}^{2,1}(r \geq 2)$ to constant flows.

The papers of Auscher, Dubois and Tchamitchian [2] and of Gallagher, Iftimie and Planchon [9] concern the stability of global regular solutions to the Navier-Stokes equations in the whole $\mathbb{R}^{3}$ with zero external force. These authors assume that appropriate norms of the solutions considered decay as $t \rightarrow \infty$.

It is worth mentioning the paper of Zhou [22], who proved the asymptotic stability of weak solutions $u \in L_{2}(0, \infty, \mathrm{BMO})$ to the Navier-Stokes equations in $\mathbb{R}^{n}, n \geq 3$, with force vanishing as $t \rightarrow \infty$.

An interesting result was obtained by Karch and Pilarczyk [11, who concentrate on the stability of Landau solutions to the Navier-Stokes system in $\mathbb{R}^{3}$. Assuming that the external force is a singular distribution they prove the asymptotic stability of the solution under any $L_{2}$-perturbation.

Paper [7] of Chemin and Gallagher is devoted to the stability of some unique global solution with large data in a very weak sense.

Finally, the stability of Leray-Hopf weak solutions has recently been examined by Bardos et al. [3], where equations with vanishing external force are considered. That paper concerns the following three cases: two-dimensional flows in infinite cylinders under three-dimensional perturbations which are periodic in the vertical direction; helical flows in circular cylinders under general three-dimensional perturbations; and axisymmetric flows under general three-dimensional perturbations. The theorem concerning the first case extends a result obtained by Gallagher [8] for purely periodic boundary conditions.

Most of the papers discussed above concern the case with zero external force [2, 3, 4, 9, 10, 16, 17], or with force which decays as $t \rightarrow \infty$ [22]. Exceptions are [11, 14, 15], where very special external forces, which are singular distributions in [11] or potentials in [14, 15], are considered. However, the case of potential forces is easily reduced to the case of zero external forces.

The aim of our paper is to prove a stability result for a large class of external forces $f_{s}$ which do not produce solutions decaying as $t \rightarrow \infty$.

It is essential that our stability results are obtained together with the existence of a global strong three-dimensional solution close to a two-dimensional one.

The paper is divided into two main parts. In the first we prove existence of global strong two-dimensional solutions not vanishing as $t \rightarrow \infty$ because the external force does not vanish either. To prove existence of such solutions 
we use the step by step method. For this purpose we have to show that the data in the time interval $[k T,(k+1) T], k \in \mathbb{N}_{0}$, do not increase with $k$. We do not need any restrictions on the time step $T$.

In the second part we prove existence of three-dimensional solutions that remain close to two-dimensional solutions. For this we need the initial velocity and the external force to be sufficiently close in apropriate norms to the initial velocity and the external force of the two-dimensional problems.

The proofs of this paper are based on the energy method, which strongly simplifies thanks to the periodic boundary conditions. The proofs of global existence which follow from the step by step technique are possible thanks to the natural decay property of the Navier-Stokes equations. This is mainly used in the first part of the paper (Section 3). To prove stability (Section 4) we use smallness of $v(0)-v_{s}(0), f-f_{s}$ and a contradiction argument applied to the nonlinear ordinary differential inequality (4.20).

We restrict ourselves to proving estimates only, because existence follows easily by the Faedo-Galerkin method.

The paper is a substantial generalization of [21] because proofs are simpler, fewer restrictions are imposed on data and there is no relation between $T, \nu$ and $f_{s}$ which in [21] implies some smallness for two-dimensional solutions.

The paper is organized as follows. In Section 2 we introduce notation and give some auxiliary results. Section 3 is devoted to the existence of a twodimensional solution. It also contains some useful estimates of the solution. In Section 4 we prove the existence of a global strong solution to problem (1.1) close to the two-dimensional solution for all time.

2. Notation and auxiliary results. We denote by $L_{p}(\Omega), p \in[1, \infty]$, the Lebesgue space of integrable functions and by $H^{s}(\Omega), s \in \mathbb{N}_{0}=\mathbb{N} \cup\{0\}$, the Sobolev space of functions with finite norm

$$
\|u\|_{H^{s}} \equiv\|u\|_{H^{s}(\Omega)}=\sum_{|\alpha| \leq s}\left(\int_{\Omega}\left|D_{x}^{\alpha} u\right|^{2} d x\right)^{1 / 2},
$$

where $D_{x}^{\alpha}=\partial_{x_{1}}^{\alpha_{1}} \partial_{x_{2}}^{\alpha_{2}} \partial_{x_{3}}^{\alpha_{3}},|\alpha|=\alpha_{1}+\alpha_{2}+\alpha_{3}, \alpha_{i} \in \mathbb{N}_{0}, i=1,2,3$.

Lemma 2.1. Assume that $f_{\Omega} f_{s}(t) d x$ and $f_{\Omega} g(t) d x$ are locally integrable on $\mathbb{R}_{+}$, and $f_{\Omega} v_{s}(0) d x$ and $f_{\Omega} u(0) d x$ are finite. Then for all $t \in \mathbb{R}_{+}$,

$$
\begin{aligned}
f_{\Omega} v_{s}(t) d x & =\int_{0}^{t} f_{\Omega} f_{s}\left(t^{\prime}\right) d x d t^{\prime}+\int_{\Omega} v_{s}(0) d x, \\
f_{\Omega} u(t) d x & =\int_{0}^{t} f_{\Omega} g\left(t^{\prime}\right) d x d t^{\prime}+f_{\Omega} u(0) d x .
\end{aligned}
$$


Proof. Applying the mean operator to (1.2) and (1.4), integrating by parts and using the periodic boundary conditions, we get (2.1) and (2.2) after integration with respect to time.

Lemma 2.2. The Poincaré inequality holds:

$$
\begin{aligned}
c_{s 1}\left\|\bar{v}_{s}\right\|_{H^{1}}^{2} & \leq\left\|\nabla \bar{v}_{s}\right\|_{L_{2}}^{2}, \\
c_{1}\|\bar{u}\|_{H^{1}}^{2} & \leq\|\nabla \bar{u}\|_{L_{2}}^{2},
\end{aligned}
$$

where $c_{s 1}, c_{1}$ are positive constants.

Let us introduce the anisotropic Lebesgue and Sobolev spaces with mixed norms, $L_{p_{1}, p_{2}}(\Omega \times(0, T))$ and $W_{p_{1}, p_{2}}^{2,1}(\Omega \times(0, T)), p_{1}, p_{2} \in(1, \infty)$, with the following norms:

$$
\begin{aligned}
\|u\|_{L_{p_{2}}\left(0, T ; L_{p_{1}}(\Omega)\right)}:= & \|u\|_{L_{p_{1}, p_{2}}(\Omega \times(0, T))} \\
:= & \left(\int_{0}^{T}\left(\int_{\Omega}|u|^{p_{1}} d x\right)^{p_{2} / p_{1}} d t\right)^{1 / p_{2}}, \\
\|u\|_{W_{p_{1}, p_{2}}^{2,1}(\Omega \times(0, T))}:= & \left\|D_{x}^{2} u\right\|_{L_{p_{1}, p_{2}}(\Omega \times(0, T))}+\left\|\partial_{t} u\right\|_{L_{p_{1}, p_{2}}(\Omega \times(0, T))} \\
& +\|u\|_{L_{p_{1}, p_{2}}(\Omega \times(0, T))} .
\end{aligned}
$$

We introduce the Besov space $B_{p, q}^{s}(\Omega)$ (see [1, Ch. 7, Sect. 7.32]) by

$$
B_{p, q}^{s}(\Omega):=\left(L_{p}(\Omega), W_{p}^{m}(\Omega)\right)_{s / m, q, J},
$$

where $s<m \in \mathbb{N}$ and $J$ is the $J$-method of interpolation (see [1, Ch. 7]). In [5. Ch. 4, Sect. 18] the Besov spaces are introduced more explicitly.

Let us consider the Stokes system

$$
\begin{array}{ll}
\omega_{t}-\nu \Delta \omega+\nabla q=f & \text { in } \Omega \times(0, T), \\
\operatorname{div} \omega=0 & \text { in } \Omega \times(0, T), \\
\left.\omega\right|_{t=0}=\omega(0) & \text { in } \Omega .
\end{array}
$$

Lemma 2.3. Let $p_{1}, p_{2} \in(1, \infty), f \in L_{p_{2}}\left(0, T ; L_{p_{1}}(\Omega)\right)$ and $\omega(0) \in$ $B_{p_{1}, p_{2}}^{2-2 / p_{2}}(\Omega)$. Then there exists a solution $(\omega, q)$ to problem $(2.5)$ such that $\omega \in W_{p_{1}, p_{2}}^{2,1}(\Omega \times(0, T)), \nabla q \in L_{p_{2}}\left(0, T ; L_{p_{1}}(\Omega)\right)$ and

$$
\begin{aligned}
\|\omega\|_{W_{p_{1}, p_{2}}^{2,1}(\Omega \times(0, T))}+ & \|\nabla q\|_{L_{p_{2}}\left(0, T ; L_{p_{1}}(\Omega)\right)} \\
& \leq c\left(\|f\|_{L_{p_{2}}\left(0, T ; L_{p_{1}}(\Omega)\right)}+\|\omega(0)\|_{B_{p_{1}, p_{2}}^{2-2 / p_{2}}(\Omega)}\right) .
\end{aligned}
$$

Proof. We use the idea of regularizer from [18, Sect. 3], where all estimates are made in Hölder spaces. Performing the estimates in Sobolev spaces with mixed norm (see [12, 13, 19, 20]) we get the assertion. 
From [6] we have

LEMMA 2.4.

(i) Let $p, p_{0} \in(1, \infty), s \in \mathbb{R}_{+}, s>2 / p_{0}$, and $u \in W_{p, p_{0}}^{s, 2}(\Omega \times(0, T))$. Then $u\left(x, t_{0}\right)=\left.u(x, t)\right|_{t=t_{0}}$ for $t_{0} \in[0, T]$ belongs to $B_{p, p_{0}}^{s-2 / p_{0}}(\Omega)$ and

$$
\left\|u\left(\cdot, t_{0}\right)\right\|_{B_{p, p_{0}}^{s-2 / p_{0}(\Omega)}} \leq c\|u\|_{W_{p, p_{0}}^{s, s / 2}(\Omega \times(0, T))},
$$

where the constant $c$ does not depend on $u$.

(ii) For $p, p_{0} \in(1, \infty), s \in \mathbb{R}_{+}, s>2 / p_{0}$, and a given $\tilde{u} \in B_{p, p_{0}}^{s-2 / p_{0}}(\Omega)$, there exists $u \in W_{p, p_{0}}^{s, s / 2}(\Omega \times(0, T))$ such that $\left.u\right|_{t=t_{0}}=\tilde{u}$ for $t_{0} \in[0, T]$ and

$$
\|u\|_{W_{p, p_{0}}^{s, s / 2}(\Omega \times(0, T))} \leq c\|\tilde{u}\|_{B_{p, p_{0}}^{s-2 / p_{0}}(\Omega)},
$$

where the constant $c$ does not depend on $u$.

3. Two-dimensional solutions. First we have

Lemma 3.1. Let $T>0$ be given. Assume that

$$
\begin{aligned}
& A_{1}^{2}:=\frac{1}{\nu c_{s 1}} \sup _{k \in \mathbb{N}_{0}} \int_{k T}^{(k+1) T}\left\|\bar{f}_{s}(t)\right\|_{L_{2}}^{2} d t<\infty, \\
& A_{2}^{2}:=\frac{A_{1}^{2}}{1-e^{-\nu c_{s} T}}+\left\|\bar{v}_{s}(0)\right\|_{L_{2}}^{2}<\infty,
\end{aligned}
$$

where $c_{s 1}$ is introduced in (2.3). Then

$$
\left\|\bar{v}_{s}(k T)\right\|_{L_{2}}^{2} \leq A_{2}^{2}
$$

and

$$
\left\|\bar{v}_{s}(t)\right\|_{L_{2}}^{2}+\nu c_{s 1} \int_{k T}^{t}\left\|\bar{v}_{s}\left(t^{\prime}\right)\right\|_{H^{1}}^{2} d t^{\prime} \leq A_{1}^{2}+A_{2}^{2}=: A_{3}^{2}
$$

for all $t \in(k T,(k+1) T]$.

Proof. Multiplying $(1.8)_{1}$ by $\bar{v}_{s}$, integrating over $\Omega$, using the periodic boundary conditions, the Poincaré inequality (2.3) and applying the Young inequality to the r.h.s. yields

$$
\frac{d}{d t}\left\|\bar{v}_{s}\right\|_{L_{2}}^{2}+\nu c_{s 1}\left\|\bar{v}_{s}\right\|_{H^{1}}^{2} \leq \frac{1}{\nu c_{s 1}}\left\|\bar{f}_{s}\right\|_{L_{2}}^{2} .
$$

Continuing, we obtain

$$
\frac{d}{d t}\left(\left\|\bar{v}_{s}\right\|_{L_{2}}^{2} e^{\nu c_{s 1} t}\right) \leq \frac{1}{\nu c_{s 1}}\left\|\bar{f}_{s}\right\|_{L_{2}}^{2} e^{\nu c_{s 1} t} .
$$

Integrating with respect to time from $k T$ to $t \in(k T,(k+1) T]$ implies

$$
\left\|\bar{v}_{s}(t)\right\|_{L_{2}}^{2} \leq \frac{1}{\nu c_{s 1}} \int_{k T}^{t}\left\|\bar{f}_{s}\left(t^{\prime}\right)\right\|_{L_{2}}^{2} d t^{\prime}+e^{-\nu c_{s 1}(t-k T)}\left\|\bar{v}_{s}(k T)\right\|_{L_{2}}^{2} .
$$


Setting $t=(k+1) T$ we get

$$
\left\|\bar{v}_{s}((k+1) T)\right\|_{L_{2}}^{2} \leq \frac{1}{\nu c_{s 1}} \int_{k T}^{(k+1) T}\left\|\bar{f}_{s}(t)\right\|_{L_{2}}^{2} d t+e^{-\nu c_{s 1} T}\left\|\bar{v}_{s}(k T)\right\|_{L_{2}}^{2} .
$$

By iteration we have

$$
\left\|\bar{v}_{s}(k T)\right\|_{L_{2}}^{2} \leq \frac{A_{1}^{2}}{1-e^{-\nu c_{s 1} T}}+e^{-\nu c_{s 1} k T}\left\|v_{s}(0)\right\|_{L_{2}}^{2} \leq A_{2}^{2} .
$$

Hence (3.1) is proved. Integrating (3.3) with respect to time from $k T$ to $t \in(k T,(k+1) T]$ and employing (3.1), we obtain (3.2).

Next we obtain an estimate for the second derivatives.

Lemma 3.2. Let the assumptions of Lemma 3.1 hold. Let $\bar{v}_{s}(0) \in H^{1}(\Omega)$. Then

$$
\left\|\bar{v}_{s x}(k T)\right\|_{L_{2}}^{2} \leq \frac{c_{s 1} A_{1}^{2}}{1-e^{-\nu c_{s 1} T}}+\left\|\bar{v}_{s x}(0)\right\|_{L_{2}}^{2}=: A_{4}^{2}
$$

and

$$
\left\|\bar{v}_{s x}(t)\right\|_{L_{2}}^{2}+\nu c_{s 1} \int_{k T}^{t}\left\|\bar{v}_{s}\left(t^{\prime}\right)\right\|_{H^{2}}^{2} d t^{\prime} \leq A_{1}^{2}+A_{4}^{2}=: A_{5}^{2}
$$

for all $t \in(k T,(k+1) T]$.

Proof. Multiplying $(1.8)_{1}$ by $-\Delta \bar{v}_{s}$, integrating over $\Omega$ and using the fact that $\bar{v}_{s}$ is divergence free yields

$$
-\int_{\Omega} \bar{v}_{s t} \cdot \Delta \bar{v}_{s} d x+\nu \int_{\Omega}\left|\Delta \bar{v}_{s}\right|^{2}=\int_{\Omega} v_{s} \cdot \nabla \bar{v}_{s} \cdot \Delta \bar{v}_{s} d x-\int_{\Omega} \bar{f}_{s} \cdot \Delta \bar{v}_{s} d x .
$$

Integrating by parts shows that the first term on the l.h.s. equals

$$
\frac{1}{2} \frac{d}{d t} \int_{\Omega}\left|\nabla \bar{v}_{s}\right|^{2} d x
$$

To examine the first term on the r.h.s. of (3.6) we use the formula

$$
\Delta \bar{v}_{s}=\left(\begin{array}{c}
-\left(\operatorname{rot} \bar{v}_{s}\right)_{, x_{2}} \\
\left(\operatorname{rot} \bar{v}_{s}\right)_{, x_{1}}
\end{array}\right) \quad \text { where } \operatorname{rot} \bar{v}_{s}=\bar{v}_{s 2, x_{1}}-\bar{v}_{s 1, x_{2}} .
$$

Then

$$
\int_{\Omega} v_{s} \cdot \nabla \bar{v}_{s} \cdot \Delta \bar{v}_{s} d x=\int_{\Omega}\left(v_{s} \cdot \nabla \bar{v}_{s 2} \operatorname{rot} \bar{v}_{s, x_{1}}-v_{s} \cdot \nabla \bar{v}_{s 1} \operatorname{rot} \bar{v}_{s, x_{2}}\right) d x=: I .
$$

Integration by parts yields

$$
\begin{aligned}
I= & -\int_{\Omega}\left(v_{s} \cdot \nabla \bar{v}_{s 2, x_{1}}-v_{s} \cdot \nabla \bar{v}_{s 1, x_{2}}\right) \operatorname{rot} \bar{v}_{s} d x \\
& -\int_{\Omega}\left(\bar{v}_{s, x_{1}} \cdot \nabla \bar{v}_{s 2}-\bar{v}_{s, x_{2}} \cdot \nabla \bar{v}_{s 1}\right) \operatorname{rot} \bar{v}_{s} d x=: I_{1}+I_{2},
\end{aligned}
$$


where

$$
I_{1}:=-\int_{\Omega} v_{s} \cdot \nabla \operatorname{rot} \bar{v}_{s} \operatorname{rot} \bar{v}_{s} d x=0
$$

and

$$
\begin{aligned}
I_{2} & :=-\int_{\Omega}\left(\bar{v}_{s 1, x_{1}} \bar{v}_{s 2, x_{1}}+\bar{v}_{s 2, x_{1}} \bar{v}_{s 2, x_{2}}-\bar{v}_{s 1, x_{2}} \bar{v}_{s 1, x_{1}}-\bar{v}_{s 2, x_{2}} \bar{v}_{s 1, x_{2}}\right) \operatorname{rot} \bar{v}_{s} d x \\
& =-\int_{\Omega} \operatorname{div} \bar{v}_{s}\left|\operatorname{rot} \bar{v}_{s}\right|^{2} d x=0 .
\end{aligned}
$$

In view of the above considerations and the Hölder and Young inequalities applied to the last term on the r.h.s. of (3.6), we obtain from (3.6) the relation

$$
\frac{d}{d t}\left\|\bar{v}_{s x}\right\|_{L_{2}}^{2}+\nu\left\|\Delta \bar{v}_{s}\right\|_{L_{2}}^{2} \leq \frac{1}{\nu}\left\|\bar{f}_{s}\right\|_{L_{2}}^{2} .
$$

Applying the Poincaré inequality (see (2.3)) yields

$$
\frac{d}{d t}\left\|\bar{v}_{s x}\right\|_{L_{2}}^{2}+\nu c_{s 1}\left\|\bar{v}_{s x}\right\|_{L_{2}}^{2} \leq \frac{1}{\nu}\left\|\bar{f}_{s}\right\|_{L_{2}}^{2}
$$

Hence

$$
\frac{d}{d t}\left(\left\|\bar{v}_{s x}\right\|_{L_{2}}^{2} e^{\nu c_{s 1} t}\right) \leq \frac{1}{\nu}\left\|\bar{f}_{s}\right\|_{L_{2}}^{2} e^{\nu c_{s 1} t}
$$

Integrating with respect to time from $k T$ to $(k+1) T$ implies

$$
\left\|\bar{v}_{s x}((k+1) T)\right\|_{L_{2}}^{2} \leq \frac{1}{\nu} \int_{k T}^{(k+1) T}\left\|\bar{f}_{s}\right\|_{L_{2}}^{2} d t+e^{-\nu c_{s 1} T}\left\|v_{s x}(k T)\right\|_{L_{2}}^{2} .
$$

Then iteration yields

$$
\left\|\bar{v}_{s x}(k T)\right\|_{L_{2}}^{2} \leq \frac{c_{s 1} A_{1}^{2}}{1-e^{-\nu c_{s 1} T}}+e^{-\nu c_{s 1} T}\left\|\bar{v}_{s x}(0)\right\|_{L_{2}}^{2} \leq A_{4}^{2} .
$$

Hence (3.4) is proved. Integrating (3.7) with respect to time from $k T$ to $t \in(k T,(k+1) T]$ and using Lemma 2.3 yields $(3.5)$.

To show stability of the two-dimensional solutions we need higher regularity of these solutions than the one proved in Lemma 3.2: we need $v_{s} \in$ $C\left(\mathbb{R}_{+} ; W_{\sigma}^{1}(\Omega)\right)$ for all $\sigma>3$. Moreover, we want to show that

$$
\left\|v_{s}(t)\right\|_{W_{\sigma}^{1}(\Omega)} \leq c
$$

where $c$ is a constant independent of time.

Finally, we do not want to apply the energy type method for higher derivatives (see [21]), because it puts stronger restrictions on the external force. Instead, we are going to apply the increasing regularity technique. This is possible because in view of Lemma 3.2 the term $\bar{v}_{s} \cdot \nabla \bar{v}_{s}$ is in $L_{2}\left(\mathbb{R}_{+} ; L_{\sigma}(\Omega)\right)$ for all $\sigma \in(1, \infty)$. As will be seen in Section 4 , we will only need to show that $\bar{v}_{s} \in C\left(\mathbb{R}_{+} ; W_{\sigma}^{1}(\Omega)\right)$ for all $\sigma>3$ (see $\left.(4.17)\right)$. 
Lemma 3.3. Let the assumptions of Lemmas 3.1 and 3.2 hold. Assume that for all $k \in \mathbb{N}_{0}$ and $\sigma>3, v_{s}(0) \in B_{\sigma, 2}^{1}(\Omega)$ and $\bar{f}_{s} \in L_{2}(k T,(k+1) T$; $\left.L_{\sigma}(\Omega)\right)$. Then $v_{s} \in C\left(\mathbb{R}_{+} ; W_{\sigma}^{1}(\Omega)\right)$ for all $\sigma>3$ and (3.8) holds.

Proof. Since $v_{s} \cdot \nabla \bar{v}_{s} \in L_{2}\left(k T,(k+1) T ; L_{\sigma}(\Omega)\right)$ for all $\sigma \in(1, \infty)$, and $\bar{f}_{s} \in L_{2}\left(k T,(k+1) T ; L_{\sigma}(\Omega)\right)$ and $\bar{v}_{s}(0) \in B_{\sigma, 2}^{1}(\Omega)$, the theory from [12, 13, 18, 19, 20, and Lemma 3.2 imply the existence of solutions to (1.8) such that $v_{s} \in W_{\sigma, 2}^{2,1}\left(\Omega \times \mathbb{R}_{+}\right)$and

$$
\left\|\bar{v}_{s}\right\|_{W_{\sigma, 2}^{2,1}(\Omega \times(0, T))} \leq c\left(A_{5}^{2}+\left\|\bar{f}_{s}\right\|_{L_{2}\left(0, T ; L_{\sigma}(\Omega)\right)}+\left\|v_{s}(0)\right\|_{B_{\sigma, 2}^{1}(\Omega)}\right) .
$$

However, we do not know how the constant $c$ depends on time. Therefore, we cannot yet claim that (3.8) holds. We have to prove (3.8) step by step in time. Let us consider the interval $(k T,(k+1) T)$. Let $\zeta=\zeta(t)$ be a smooth cut-off function such that $\zeta(t)=0$ for $t \in[k T, k T+\delta / 2]$ and $\zeta(t)=1$ for $t \geq k T+\delta$, for some $\delta \in(T / 2, T)$. Introducing the new functions

$$
\tilde{v}_{s}=\bar{v}_{s} \zeta, \quad \tilde{p}_{s}=\bar{p}_{s} \zeta, \quad \tilde{f}_{s}=\bar{f}_{s} \zeta, \quad \dot{\zeta}=\zeta_{, t}
$$

we see that $\left(\tilde{v}_{s}, \tilde{p}_{s}\right)$ is a solution to the problem

$$
\begin{array}{ll}
\tilde{v}_{s t}-\nu \Delta \tilde{v}_{s}+\nabla \tilde{p}_{s}=\bar{v}_{s} \dot{\zeta}-v_{s} \cdot \nabla \tilde{v}_{s}+\tilde{f}_{s} & \text { in } \Omega \times(k T,(k+1) T), \\
\operatorname{div} \tilde{v}_{s}=0 & \text { in } \Omega \times(k T,(k+1) T), \\
\left.\tilde{v}_{s}\right|_{t=k T}=0 . &
\end{array}
$$

In view of Lemma 3.2 and [12, 13, 18, 19, 20] we have the existence of solutions to (3.10) such that

$$
\tilde{v}_{s} \in W_{\sigma, 2}^{2,1}(k T+\delta,(k+1) T ; \Omega), \quad \nabla \tilde{p}_{s} \in L_{\sigma, 2}(k T+\delta,(k+1) T ; \Omega)
$$

and

$$
\left\|\tilde{v}_{s}\right\|_{W_{\sigma, 2}^{2,1}(k T+\delta,(k+1) T ; \Omega)} \leq c\left(\frac{1}{\delta} A_{5}+A_{5}^{2}+\left\|\tilde{f}_{s}\right\|_{L_{2}(k T+\delta / 2,(k+1) T ; \Omega)}\right),
$$

where $c$ may depend on $T$ but it does not depend on $k$. Hence by imbedding for $\sigma>3$ estimate (3.11) implies (3.8).

To get (3.8) we only need an estimate for the interval $(k T, k T+\delta), k \in \mathbb{N}$, because for $k=0$ we have (3.9). From (3.11) with $k$ replaced by $k-1$ we obtain

$$
\left\|\tilde{v}_{s}\right\|_{W_{\sigma, 2}^{2,1}((k-1) T+\delta, k T ; \Omega)} \leq c\left(\frac{1}{\delta} A_{5}+A_{5}^{2}+\left\|\tilde{f}_{s}\right\|_{L_{2}\left((k-1)^{T}+\delta / 2, k T ; \Omega\right)}\right),
$$

so by the trace theorem (see Lemma 2.3) we derive

$$
\left\|\tilde{v}_{s}(k T)\right\|_{B_{\sigma, 2}^{1}(\Omega)} \leq c\left\|\bar{v}_{s}\right\|_{W_{\sigma, 2}^{2,1}((k-1) T+\delta, k T ; \Omega)} .
$$

Hence, repeating the considerations leading to (3.9) for the time interval 
$(k T, k T+\delta)$ we find that $\tilde{v}_{s} \in W_{\sigma, 2}^{2,1}(\Omega \times(k T, k T+\delta))$ and

$$
\begin{aligned}
& \left\|\tilde{v}_{s}\right\|_{W_{\sigma, 2}^{2,1}(\Omega \times(k T, k T+\delta))} \\
& \quad \leq c\left(A_{5}^{2}+\left\|\bar{f}_{s}\right\|_{L_{\sigma, 2}(\Omega \times(k T, k T+\delta))}+\left\|\bar{v}_{s}(k T)\right\|_{B_{\sigma, 2}^{1}(\Omega)}\right) .
\end{aligned}
$$

Hence (3.8) holds for all $t \in \mathbb{R}_{+}$and Lemma 3.3 is proved.

4. Stability. In this section we examine problem (1.4). First we derive a global estimate for the $L_{2}$ norm of $u$. We show how the restriction from assumption (2) of Lemma 4.1 appears (it is much more restrictive than in [21]). Fortunately, we do not need Lemma 4.1 to prove stability.

Lemma 4.1. Let the assumptions of Lemmas 3.1 and 3.2 hold.

(1) Set

$B_{1}^{2}:=\sup _{k \in \mathbb{N}_{0}} \int_{k T}^{(k+1) T}\left(\frac{\nu c_{1}}{2 c_{3}}\left|\int_{0}^{t} f_{\Omega} g\left(t^{\prime}\right) d x d t^{\prime}+\int_{\Omega} u(0) d x\right|^{2}+\frac{2 c_{3}}{\nu c_{1}}\|\bar{g}(t)\|_{L_{6 / 5}}^{2}\right) d t$,

where $c_{1}$ comes from the Poincaré inequality (2.4) and $c_{3}$ from the imbedding (4.3).

(2) Assume

$$
\frac{-\nu c_{1}}{2} T+\frac{4 c_{3}}{\nu c_{1}} A_{3}^{2} \leq 0
$$

(3) Set

$$
B_{2}^{2}:=\exp \left(\frac{4 c_{3}}{\nu c_{1}} A_{3}^{2}\right) B_{1}^{2}
$$

Then

$$
\begin{aligned}
\|\bar{u}(k T)\|_{L_{2}}^{2} & \leq \frac{B_{2}^{2}}{1-\exp \left(-\nu c_{1} T / 2\right)}+\|\bar{u}(0)\|_{L_{2}}^{2}=: B_{3}^{2}, \\
\|\bar{u}(t)\|_{L_{2}}^{2} & \leq B_{2}^{2}+B_{3}^{2}=: B_{4}^{2},
\end{aligned}
$$

for $t \in[k T,(k+1) T]$ and any $k \in \mathbb{N}_{0}$.

Proof. Multiplying (1.9) 1 by $\bar{u}$ and integrating over $\Omega$ gives

$$
\begin{aligned}
& \frac{1}{2} \frac{d}{d t}\|\bar{u}\|_{L_{2}}^{2}+\nu c_{1}\left\|\bar{u}_{x}\right\|_{L_{2}}^{2} \leq\left|\int_{\Omega} u \cdot \nabla \bar{v}_{s} \cdot \bar{u} d x\right|+\left|\int_{\Omega} \bar{g} \cdot \bar{u} d x\right| \\
& \leq\left|\int_{\Omega} \bar{u} \cdot \nabla \bar{v}_{s} \cdot \bar{u} d x\right|+\left|f_{\Omega} u d x \cdot \int_{\Omega} \nabla \bar{v}_{s} \cdot \bar{u} d x\right|+\left|\int_{\Omega} \bar{g} \cdot \bar{u} d x\right| .
\end{aligned}
$$

Employing the estimates

$$
\left|\int_{\Omega} \bar{u} \cdot \nabla \bar{v}_{s} \cdot \bar{u} d x\right| \leq \frac{\varepsilon_{1}}{2}\|\bar{u}\|_{L_{6}}^{2}+\frac{1}{2 \varepsilon_{1}}\left\|\bar{v}_{s x}\right\|_{L_{3}}^{2}\|\bar{u}\|_{L_{2}}^{2},
$$




$$
\begin{aligned}
& \left|f u d x \cdot \int_{\Omega} \nabla \bar{v}_{s} \cdot \bar{u} d x\right|=\frac{1}{2 \varepsilon_{2}}\left\|\nabla \bar{v}_{s}\right\|_{L_{2}}^{2}\|\bar{u}\|_{L_{2}}^{2}+\frac{\varepsilon_{2}}{2}|f u d x|^{2}, \\
& \left|\int_{\omega} \bar{g} \cdot \bar{u} d x\right| \leq \frac{\varepsilon_{3}}{2}\|\bar{u}\|_{L_{6}}^{2}+\frac{1}{2 \varepsilon_{3}}\|\bar{g}\|_{L_{6 / 5}}^{2},
\end{aligned}
$$

for all $\varepsilon_{1}, \varepsilon_{2}, \varepsilon_{3}>0$, together with (2.4) and the imbedding

$$
\|\bar{u}\|_{L_{6}}^{2} \leq c_{3}\|\bar{u}\|_{H^{1}}^{2},
$$

and assuming that $\left(\varepsilon_{i} / 2\right) c_{3} \leq \nu c_{1} / 4, i=1,3$, we obtain from (4.2) the inequality

$$
\begin{aligned}
\frac{1}{2} \frac{d}{d t}\|\bar{u}\|_{L_{2}}^{2}+\frac{\nu c_{1}}{2}\|\bar{u}\|_{H^{1}}^{2} \leq & \frac{c_{3}}{\nu c_{1}}\left(\left\|\bar{v}_{s x}\right\|_{L_{3}}^{2}+\left\|\bar{v}_{s x}\right\|_{L_{2}}^{2}\right)\|\bar{u}\|_{L_{2}}^{2} \\
& +\frac{\nu c_{1}}{4 c_{3}}|f u d x|^{2}+\frac{c_{3}}{\nu c_{1}}\|\bar{g}\|_{L_{6 / 5}}^{2},
\end{aligned}
$$

where we have set $\varepsilon_{2}:=\frac{\nu c_{1}}{2 c_{3}}$. Employing (2.2) in (4.4) yields

$$
\begin{aligned}
\frac{d}{d t}\|\bar{u}\|_{L_{2}}^{2}+\nu c_{1}\|\bar{u}\|_{H^{1}}^{2} & \leq \frac{2 c_{3}}{\nu c_{1}}\left(\left\|\bar{v}_{s x}\right\|_{L_{3}}^{2}+\left\|\bar{v}_{s x}\right\|_{L_{2}}^{2}\right)\|\bar{u}\|_{L_{2}}^{2} \\
& +\frac{\nu c_{1}}{2 c_{3}}\left|\int_{0}^{t} f_{\Omega} g\left(t^{\prime}\right) d x d t^{\prime}+f_{\Omega} u(0) d x\right|^{2}+\frac{2 c_{3}}{\nu c_{1}}\|\bar{g}\|_{L_{6 / 5}}^{2} .
\end{aligned}
$$

Considering (4.5) for $t \in(k T,(k+1) T)$ we have

$$
\begin{aligned}
\frac{d}{d t}\left[\|\bar{u}(t)\|_{L_{2}}^{2} \exp \left(\nu c_{1} t-\frac{2 c_{3}}{\nu c_{1}} \int_{k T}^{t}\left(\left\|\bar{v}_{s x}\left(t^{\prime}\right)\right\|_{L_{3}}^{2}+\left\|\bar{v}_{s x}\left(t^{\prime}\right)\right\|_{L_{2}}^{2}\right) d t^{\prime}\right)\right] \\
\leq\left(\frac{\nu c_{1}}{2 c_{3}}\left|\int_{0}^{t} f_{\Omega} g\left(t^{\prime}\right) d x d t^{\prime}+\int_{\Omega} u(0) d x\right|^{2}+\frac{2 c_{3}}{\nu c_{1}}\|\bar{g}(t)\|_{L_{6 / 5}}^{2}\right) \\
\cdot \exp \left(\nu c_{1} t-\frac{2 c_{3}}{\nu c_{1}} \int_{k T}^{t}\left(\left\|\bar{v}_{s x}\left(t^{\prime}\right)\right\|_{L_{3}}^{2}+\left\|\bar{v}_{s x}\left(t^{\prime}\right)\right\|_{L_{2}}^{2}\right) d t^{\prime}\right) .
\end{aligned}
$$

Integrating (4.6) with respect to time from $k T$ to $t \in(k T,(k+1) T]$ implies

$$
\begin{aligned}
\|\bar{u}(t)\|_{L_{2}}^{2} \leq \exp \left[\frac{2 c_{3}}{\nu c_{1}} \int_{k T}^{t}\left(\left\|v_{s x}\left(t^{\prime}\right)\right\|_{L_{3}}^{2}+\left\|v_{s x}\left(t^{\prime}\right)\right\|_{L_{2}}^{2}\right) d t^{\prime}\right] . \\
\cdot \int_{k T}^{t}\left(\frac{\nu c_{1}}{2 c_{3}}\left|\int_{0}^{t^{\prime}} \int_{\Omega} g\left(t^{\prime \prime}\right) d x d t^{\prime \prime}+\int_{\Omega} u(0) d x\right|^{2}+\frac{2 c_{3}}{\nu c_{1}}\left\|\bar{g}\left(t^{\prime}\right)\right\|_{L_{6 / 5}}^{2}\right) d t^{\prime} \\
+\|\bar{u}(k T)\|_{L_{2}}^{2} \exp \left[-\nu c_{1}(t-k T)+\frac{2 c_{3}}{\nu c_{1}} \int_{k T}^{t}\left(\left\|\bar{v}_{s x}\left(t^{\prime}\right)\right\|_{L_{3}}^{2}+\left\|\bar{v}_{s x}\left(t^{\prime}\right)\right\|_{L_{2}}^{2}\right) d t^{\prime}\right] .
\end{aligned}
$$


Setting $t=(k+1) T$ in (4.7) and using (3.2) yields

$$
\begin{aligned}
&\|\bar{u}((k+1) T)\|_{L_{2}}^{2} \leq \exp \left(\frac{4 c_{3}}{\nu c_{1}} A_{3}^{2}\right) \\
& \times \int_{k T}^{(k+1) T}\left[\frac{\nu c_{1}}{2 c_{3}}\left|\int_{0}^{t} f_{\Omega} g\left(t^{\prime}\right) d x d t^{\prime}+\int_{\Omega} u(0) d x\right|^{2}+\frac{2 c_{3}}{c_{1}}\|\bar{g}(t)\|_{L_{6 / 5}}^{2}\right] d t \\
&+\|\bar{u}(k T)\|_{L_{2}}^{2} \exp \left(-\nu c_{1} T+\frac{4 c_{3}}{\nu c_{1}} A_{3}^{2}\right) .
\end{aligned}
$$

In view of assumptions (1)-(3) of the lemma we have

$$
\|\bar{u}((k+1) T)\|_{L_{2}}^{2} \leq B_{2}^{2}+\exp \left(\frac{-\nu c_{1}}{2} T\right)\|\bar{u}(k T)\|_{L_{2}}^{2} .
$$

Iteration implies

$$
\|\bar{u}(k T)\|_{L_{2}}^{2} \leq \frac{B_{2}^{2}}{1-\exp \left(-\nu c_{1} T / 2\right)}+\exp \left(\frac{-\nu c_{1}}{2} k T\right)\|\bar{u}(0)\|_{L_{2}}^{2} .
$$

Hence $(4.1)_{1}$ is proved. Employing the assumptions of the lemma and $(4.1)_{1}$ in (4.7) gives $(4.1)_{2}$.

REMARK 4.2. Assumption (2) of Lemma 4.1 has the explicit form

$$
\frac{2-\exp \left(-\nu c_{s 1} T\right)}{c_{s 1} \nu\left(1-\exp \left(-\nu c_{s 1} T\right)\right)} \sup _{k \in \mathbb{N}_{0}} \int_{k T}^{(k+1) T}\left\|\bar{f}_{s}(t)\right\|_{L_{2}}^{2} d t+\left\|\bar{v}_{s x}(0)\right\|_{L_{2}}^{2} \leq \frac{\nu^{2} c_{1}^{2}}{8 c_{3}} T .
$$

Assuming that $\left\|\bar{v}_{s x}(0)\right\|_{L_{2}}$ is given we see that (4.11) holds for

$$
T>\frac{8 c_{3}}{\nu^{2} c_{1}^{2}}\left\|\bar{v}_{s x}(0)\right\|_{L_{2}}^{2} .
$$

For such large $T$ we have a strong restriction on $\sup _{k \in \mathbb{N}_{0}} \int_{k T}^{(k+1) T}\left\|\bar{f}_{s}(t)\right\|_{L_{2}}^{2} d t$. Physically, this means that the energy introduced to the region under consideration should not be too large compared with the dissipation.

Finally, we show that $3 \mathrm{~d}$ solutions to (1.1) remain close to $2 \mathrm{~d}$ solutions to (1.2) for all time if their initial data and the external forces are sufficiently close. In this proof we omit the heavy restriction (4.11).

Lemma 4.3. Assume $\bar{v}_{s} \in C\left(\mathbb{R}_{+} ; W_{3}^{1}\right), \bar{g} \in C\left(\mathbb{R}_{+} ; L_{2}\right)$ and $\bar{u}(0) \in H^{1}$. Let $\gamma \in\left(0, \gamma_{*}\right]$, where $\nu c_{4}-\frac{c_{5}}{\nu^{3}} \gamma_{*}^{2} \geq \frac{c_{*}}{2}, c_{*}<\nu c_{4}$ and $c_{4}, c_{5}$ are introduced in (4.16). Assume that

$$
\begin{aligned}
& \|\bar{u}(0)\|_{H^{1}}^{2} \leq \gamma \\
& G^{2}(t):=\frac{c_{5}}{\nu}\left[\left\|\bar{v}_{s x}\right\|_{L_{3}}^{2}\left|\int_{0}^{t} \int_{\Omega} g\left(x, t^{\prime}\right) d x d t^{\prime}+\int_{\Omega} u(0) d x\right|^{2}+\|\bar{g}\|_{L_{2}}^{2}\right] \leq c_{*} \frac{\gamma}{4} .
\end{aligned}
$$


Let $T>0$ be given and $k \in \mathbb{N}_{0}$. Assume that

$$
\begin{aligned}
& \frac{c_{5}}{\nu} \int_{k T}^{(k+1) T}\left\|\bar{v}_{s x}\right\|_{L_{3}}^{2} d t \leq \frac{c_{*}}{4} T, \quad \int_{k T}^{(k+1) T} G^{2}(t) d t \leq \alpha \gamma, \\
& \alpha \exp \left(\frac{c_{*}}{4} T\right)+\exp \left(-\frac{c_{*}}{4} T\right) \leq 1 .
\end{aligned}
$$

Then

$$
\|\bar{u}(t)\|_{H^{1}}^{2} \leq \gamma \quad \text { for } t \in \mathbb{R}_{+} .
$$

Proof. Differentiating $(1.9)_{1}$ with respect to $x$, multiplying the result by $\bar{u}_{x}$, integrating over $\Omega$ and employing the periodic boundary conditions yields

$$
\begin{aligned}
\frac{1}{2} \frac{d}{d t}\left\|\bar{u}_{x}\right\|_{L_{2}}^{2}+\nu\left\|\bar{u}_{x x}\right\|_{L_{2}}^{2} \leq & \left\|\bar{u}_{x}\right\|_{L_{3}}^{3}+\left|\int_{\Omega} \bar{v}_{s x} \cdot \nabla \bar{u} \cdot \bar{u}_{x} d x\right| \\
& +\left|\int_{\Omega} u \cdot \nabla \bar{v}_{s} \cdot \bar{u}_{x x} d x\right|+\left|\int_{\Omega} \bar{g} \cdot \bar{u}_{x x} d x\right| .
\end{aligned}
$$

Adding (4.2) and (4.14), and applying the Hölder, Young and Poincaré inequalities, we derive

$$
\begin{aligned}
& \frac{d}{d t}\|\bar{u}\|_{H^{1}}^{2}+\nu c\|\bar{u}\|_{H^{2}}^{2} \\
& \quad \leq c\left(\left\|\bar{u}_{x}\right\|_{L_{3}}^{3}+\frac{1}{\nu}\left\|\bar{v}_{s x}\right\|_{L_{3}}^{2}\left\|\bar{u}_{x}\right\|_{L_{2}}^{2}+\frac{1}{\nu}\|u\|_{L_{6}}^{2}\left\|\bar{v}_{s x}\right\|_{L_{3}}^{2}+\frac{1}{\nu}\|\bar{g}\|_{L_{2}}^{2}\right) .
\end{aligned}
$$

Using $\|u\|_{L_{6}}^{2} \leq c\left(\|\bar{u}\|_{L_{6}}^{2}+\left|f_{\Omega} u d x\right|^{2}\right)$ and $\|\bar{u}\|_{L_{6}} \leq c\|\bar{u}\|_{H^{1}} \leq c\left\|\bar{u}_{x}\right\|_{L_{2}}$, which holds in view of the Poincaré inequality, we get

$$
\begin{aligned}
\frac{d}{d t}\|\bar{u}\|_{H^{1}}^{2} & +\nu c\|\bar{u}\|_{H^{2}}^{2} \\
& \leq c\left[\left\|\bar{u}_{x}\right\|_{L_{3}}^{3}+\frac{1}{\nu}\left\|\bar{v}_{s x}\right\|_{L_{3}}^{2}\left(\left\|\bar{u}_{x}\right\|_{L_{2}}^{2}+\left|f_{\Omega} u d x\right|^{2}\right)+\frac{1}{\nu}\|\bar{g}\|_{L_{2}}^{2}\right] .
\end{aligned}
$$

In view of (2.2) and the interpolation inequality (see [5, Ch. 3, Sect. 15])

$$
\left\|\bar{u}_{x}\right\|_{L_{3}} \leq c\left\|\bar{u}_{x x}\right\|_{L_{2}}^{1 / 2}\left\|\bar{u}_{x}\right\|_{L_{2}}^{1 / 2}
$$

(which holds without the lower order term because $\int_{\Omega} \bar{u}_{x} d x=0$ ), we obtain from (4.15) the inequality

$$
\begin{aligned}
\frac{d}{d t}\|\bar{u}\|_{H^{1}}^{2}+ & \nu c_{4}\|\bar{u}\|_{H^{2}}^{2} \leq \frac{c_{5}}{\nu^{3}}\left\|\bar{u}_{x}\right\|_{L_{2}}^{6}+\frac{c_{5}}{\nu}\left\|\bar{v}_{s x}\right\|_{L_{3}}^{2}\left\|\bar{u}_{x}\right\|_{L_{2}}^{2} \\
& +\frac{c_{5}}{\nu}\left\|\bar{v}_{s x}\right\|_{L_{3}}^{2}\left|\int_{0}^{t} \int_{\Omega} g\left(x, t^{\prime}\right) d x d t^{\prime}+\int_{\Omega} u(0) d x\right|^{2}+\frac{c_{5}}{\nu}\|\bar{g}\|_{L_{2}}^{2} .
\end{aligned}
$$


To prove the lemma we need to know that the r.h.s. of (4.16) is bounded. We consider (4.16) in the time interval $(k T,(k+1) T), k \in \mathbb{N}_{0}$. Assume that we have proved that $u(k T) \in H^{1}(\Omega)$ and $\|u(k T)\|_{H^{1}}^{2} \leq \gamma$, where $\gamma$ is sufficiently small. Using that $\bar{g} \in L_{2}(\Omega \times(k T,(k+1) T))$ is sufficiently small we have existence of solutions to problem (1.9) in $W_{2}^{2,1}(\Omega \times(k T,(k+1) T))$ because the other terms on the r.h.s. of (1.9) also belong to $L_{2}(\Omega \times(k T$, $(k+1) T))$ in view of imbeddings and the assumption that $v_{s} \in W_{2}^{2,1}(\Omega \times(k T$, $(k+1) T)$ ). The last assertion holds in view of the assumptions of Lemma 3.2 and the restriction that $v_{s}$ is a two-dimensional solution to the Navier-Stokes equations. However, to have the r.h.s. of (4.16) bounded we need that $v_{s} \in$ $L_{\infty}\left(k T,(k+1) T ; W_{3^{+}}^{1}(\Omega)\right)$, where $3^{+}>3$ but is close to 3 . This follows from Lemma 3.3, where it is proved that $v_{s} \in W_{\sigma, 2}^{2,1}(\Omega \times(k T,(k+1) T))$ for any $\sigma>3$ if the data are sufficiently smooth.

In view of the above remarks we can introduce the quantities

$$
\begin{aligned}
G^{2}(t) & :=\frac{c_{5}}{\nu}\left(\left\|\bar{v}_{s x}\right\|_{L_{3}}^{2}\left|\int_{0}^{t} f_{\Omega} g\left(x, t^{\prime}\right) d x d t^{\prime}+\int_{\Omega} u(0) d x\right|^{2}+\frac{1}{-}\|\bar{g}\|_{L_{2}}^{2}\right), \\
A^{2}(t) & :=\frac{c_{5}}{\nu}\left\|\bar{v}_{s x}\right\|_{L_{3}}^{2}, \\
X(t) & :=\|\bar{u}(t)\|_{H^{1}}, \quad Y(t)=\|\bar{u}(t)\|_{H^{2}} .
\end{aligned}
$$

Then (4.16) takes the form

$$
\frac{d}{d t} X^{2}+\nu c_{4} Y^{2} \leq \frac{c_{5}}{\nu^{3}} X^{4} X^{2}+A^{2} X^{2}+G^{2} .
$$

Since $X \leq Y$ we have

$$
\frac{d}{d t} X^{2} \leq-X^{2}\left(\nu c_{4}-\frac{c_{5}}{\nu^{3}} X^{4}\right)+A^{2} X^{2}+G^{2} .
$$

Let $\gamma \in\left(0, \gamma_{*}\right]$, where $\gamma_{*}$ is so small that

$$
\nu c_{4}-\frac{c_{5}}{\nu^{3}} \gamma_{*}^{2} \geq c_{*} / 2, \quad c_{*}<\nu c_{4} .
$$

Since the coefficients of (4.18) depend on the two-dimensional solution determined step by step in time, we consider (4.18) in the interval $[k T,(k+1) T]$, $k \in \mathbb{N}_{0}$, with the assumptions

$$
X^{2}(k T) \leq \gamma, \quad G^{2}(t) \leq c_{*} \gamma / 4 \quad \text { for all } t \in[k T,(k+1) T] .
$$

Let us introduce the quantity

$$
Z^{2}(t):=\exp \left(-\int_{k T}^{t} A^{2}\left(t^{\prime}\right) d t^{\prime}\right) X^{2}(t), \quad t \in[k T,(k+1) T] .
$$


Then (4.18) takes the form

$$
\frac{d}{d t} Z^{2} \leq-\left(\nu c_{4}-\frac{c_{5}}{\nu^{3}} X^{4}\right) Z^{2}+\bar{G}^{2},
$$

where $\bar{G}^{2}:=G^{2} \exp \left(-\int_{k T}^{t} A^{2}\left(t^{\prime}\right) d t^{\prime}\right)$.

Suppose that

$$
\begin{aligned}
t_{*} & :=\inf \left\{t \in(k T,(k+1) T]: X^{2}(t)>\gamma\right\} \\
& =\inf \left\{t \in(k T,(k+1) T]: Z^{2}(t)>\gamma \exp \left(-\int_{k T}^{t} A^{2}\left(t^{\prime}\right) d t^{\prime}\right)\right\}>k T .
\end{aligned}
$$

By (4.19) for $t \in\left(0, t_{*}\right]$ inequality (4.20) takes the form

$$
\frac{d}{d t} Z^{2} \leq-\frac{c_{*}}{2} Z^{2}+\bar{G}^{2}(t) .
$$

Clearly, we have

$$
\begin{aligned}
& Z^{2}\left(t_{*}\right)=\gamma \exp \left(-\int_{k T}^{t_{*}} A^{2}\left(t^{\prime}\right) d t^{\prime}\right) \\
& Z^{2}(t)>\gamma \exp \left(-\int_{k T}^{t_{*}} A^{2}\left(t^{\prime}\right) d t^{\prime}\right) \quad \text { for } t>t_{*} .
\end{aligned}
$$

Then (4.21) yields

$$
\left.\frac{d}{d t} Z^{2}\right|_{t=t_{*}} \leq c_{*}\left(-\frac{\gamma}{2}+\frac{\gamma}{4}\right) \exp \left(-\int_{k T}^{t_{*}} A^{2}\left(t^{\prime}\right) d t^{\prime}\right)<0,
$$

contradicting (4.22). Therefore

$$
Z^{2}(t)<\gamma \exp \left(-\int_{k T}^{t_{*}} A^{2}\left(t^{\prime}\right) d t^{\prime}\right) \quad \text { for } t>t_{*} .
$$

The definition of $Z^{2}(t)$ implies

$$
X^{2}(t) \leq \gamma \exp \left(\int_{t_{*}}^{t} A^{2}\left(t^{\prime}\right) d t^{\prime}\right) \quad \text { for } t>t_{*} .
$$

For sufficiently small $\gamma$ inequality (4.18) takes the form

$$
\frac{d}{d t} X^{2}+\frac{c_{*}}{2} X^{2} \leq A^{2} X^{2}+G^{2} .
$$

Integrating (4.24) with respect to time from $t=k T$ to $t=(k+1) T$ gives 


$$
\begin{aligned}
X^{2}((k+1) T) \leq & \exp \left(\int_{k T}^{(k+1) T} A^{2}(t) d t\right) \int_{k T}^{(k+1) T} G^{2}(t) d t \\
& +\exp \left(-\frac{c_{*}}{2} T+\int_{k T}^{(k+1) T} A^{2}(t) d t\right) X^{2}(k T) .
\end{aligned}
$$

In view of the assumptions

$$
\frac{c_{*}}{4} T \geq \int_{k T}^{(k+1) T} A^{2}(t) d t, \quad \int_{k T}^{(k+1) T} G^{2}(t) d t \leq \alpha \gamma,
$$

where $\alpha$ is so small and $T$ so large that

$$
\alpha \exp \left(\int_{k T}^{(k+1) T} A^{2}(t) d t\right)+\exp \left(-\frac{c_{*}}{4} T\right) \leq 1,
$$

we find that $X^{2}((k+1) T)<\gamma$. Then by induction we obtain the lemma.

Acknowledgements. The author thanks Professor Y. Shibata for important comments concerning the proof of Lemma 4.3.

The research leading to these results received funding from the People Programme (Marie Curie Actions) of the European Union's Seventh Framework Programme FP7/2007-2013/ under REA grant agreement $\mathrm{n}^{\circ} 319012$ and from the Funds for International Co-operation under Polish Ministry of Science and Higher Education grant agreement n ${ }^{\circ}$ 2853/7.PR/2013/2.

\section{References}

[1] R. A. Adams and J. J. F. Fournier, Sobolev Spaces, 2nd ed., Academic Press, 2008.

[2] P. Auscher, S. Dubois and P. Tchamitchian, On the stability of global solutions to Navier-Stokes equations in the space, J. Math. Pures Appl. 83 (2004), 673-697.

[3] C. Bardos, M. C. Lopes Filho, D. Niu, H. J. Nussenzveig Lopes and E. S. Titi, Stability of two-dimensional viscous incompressible flows under three-dimensional perturbations and inviscid symmetry breaking, SIAM J. Math. Anal. 45 (2013), 1871-1885.

[4] H. Beirão da Veiga and P. Secchi, $L^{p}$-stability for the strong solutions of the NavierStokes equations in the whole space, Arch. Ration. Mech. Anal. 98 (1987), 65-69.

[5] O. V. Besov, V. P. Il'in and S. M. Nikol'skiǔ, Integral Representations of Functions and Imbedding Theorems, Nauka, Moscow, 1975 (in Russian).

[6] Ya. S. Bugrov, Function spaces with mixed norm, Math. USSR-Izv. 5 (1971), 11451167.

[7] J. I. Chemin and I. Gallagher, Wellposedness and stability results for the NavierStokes equations in $\mathbb{R}^{3}$, Ann. Inst. H. Poincaré Anal. Non Linéaire 26 (2009), 599-624.

[8] I. Gallagher, The tridimensional Navier-Stokes equations with almost bidimensional data: stability, uniqueness and life span, Int. Mat. Res. Notices 1997, 919-935.

[9] I. Gallagher, D. Iftimie and F. Planchon, Asymptotics and stability for global solutions to the Navier-Stokes equations, Ann. Inst. Fourier (Grenoble) 53 (2003), 1387-1424. 
[10] D. Iftimie, The 3D Navier-Stokes equations seen as a perturbation of the 2D NavierStokes equations, Bull. Soc. Math. France 127 (1999), 473-517.

[11] G. Karch and D. Pilarczyk, Asymptotic stability of Landau solutions to Navier-Stokes system, Arch. Ration. Mech. Anal. 202 (2011), 115-131.

[12] N. V. Krylov, The heat equation in $L_{q}\left((0, T) ; L_{p}\right)$-spaces with weights, SIAM J. Math. Anal. 32 (2001), 1117-1141.

[13] N. V. Krylov, The Calderón-Zygmund theorem and its applications to parabolic equations, Algebra i Analiz 13 (2001), no. 4, 1-25 (in Russian).

[14] P. B. Mucha, Stability of nontrivial solutions of the Navier-Stokes system on the three dimensional torus, J. Differential Equations 172 (2001), 359-375.

[15] P. B. Mucha, Stability of constant solutions to the Navier-Stokes system in $\mathbb{R}^{3}$, Appl. Math. (Warsaw) 28 (2001), 301-310.

[16] P. B. Mucha, Stability of $2 D$ incompressible flows in $\mathbb{R}^{3}$, J. Differential Equations 245 (2008), 2355-2367.

[17] G. Ponce, R. Racke, T. C. Sideris and E. S. Titi, Global stability of large solutions to the 3d Navier-Stokes equations, Comm. Math. Phys. 159 (1994), 329-341.

[18] V. A. Solonnikov, On the solvability of generalized Stokes equations in the spaces of periodic functions, Ann. Univ. Ferrara Sez. VII 46 (2000), 219-249.

[19] V. A. Solonnikov, Estimates of solutions of the Stokes equations in Sobolev spaces with a mixed norm, Zap. Nauchn. Sem. POMI 288 (2002), 204-231 (in Russian).

[20] V. A. Solonnikov, Estimates of solutions of the nonstationary Stokes problem in anisotropic Sobolev spaces and estimates for the resolvent of the Stokes operator, Uspekhi Mat. Nauk 58 (2003), no. 2, 123-156 (in Russian).

[21] E. Zadrzyńska and W. M. Zajączkowski, Stability of two-dimensional Navier-Stokes motions in the periodic case, J. Math. Anal. Appl. 423 (2015), 956-974.

[22] Y. Zhou, Asymptotic stability for the Navier-Stokes equations in the marginal class, Proc. Roy. Soc. Edinburgh 136 (2006), 1099-1109.

W. M. Zajączkowski

Institute of Mathematics

Polish Academy of Sciences

Śniadeckich 8

00-656 Warszawa, Poland

and

Institute of Mathematics and Cryptology

Cybernetics Faculty

Military University of Technology

Kaliskiego 2

00-908 Warszawa, Poland

E-mail:wz@impan.pl 
